\title{
Liver failure due to relapsed myeloma and hepatic iron overload
}

\author{
Chakra P Chaulagain ${ }^{1}$, Adalberto Gonzalez ${ }^{2}$, Isaac Goldszer ${ }^{3}$, Luis Caraballo ${ }^{4}$, James E Hoffman ${ }^{5}$, Leah Elson ${ }^{1}$ and Maria-Julia Diacovo ${ }^{6}$ \\ ${ }^{1}$ Department of Hematology and Oncology, Maroone Cancer Center, Myeloma and Amyloidosis Program, Cleveland Clinic Florida, Weston, Florida, USA \\ ${ }^{2}$ Department of Gastroenterology and Hepatology, Cleveland Clinic Florida, Weston, Florida, USA \\ ${ }^{3}$ Department of Neurology, Medical University of South Carolina, Charleston, SC, USA \\ ${ }^{4}$ Department of internal medicine, Kendall Regional Medical Center, Kendall FL, USA \\ ${ }^{5}$ Sylvester Comprehensive Cancer Center at University of Miami, Amyloidosis and Myeloma Program, Miami, USA \\ ${ }^{6}$ Department of Pathology, Cleveland Clinic Florida, Weston, Florida, USA
}

\section{Abstract}

Multiple myeloma is a hematologic malignancy that classically manifests with hypercalcaemia, renal insufficiency, anaemia and lytic bone lesions. Liver dysfunction in multiple myeloma is a lesser known complication that occurs through biliary obstruction, liver infiltration by plasma cells, amyloid/light chain deposition or due to liver injury from medications. Although transfusion-related hepatic iron overload-leading to significant liver disease-is a recognised complication in certain hematologic malignancies, little is known about transfusional iron overload in patients with multiple myeloma. We present a case of a 49-year-old female with relapsed/refractory multiple myeloma who presented with rapid onset liver failure, due to both iron deposition and malignant plasma cell infiltration of the liver as a terminal event. A review of the literature on hepatic complications in multiple myeloma patients is presented.

Keywords: multiple myeloma, iron overload, liver dysfunction, liver failure

\section{Introduction}

Multiple myeloma (MM) is an incurable hematologic malignancy of plasma cells. It can present with hypercalcaemia, renal failure, anaemia and/or lytic bone lesions or fractures. In general, liver involvement in myeloma is a rare event. However, direct infiltration of the liver leading to intrahepatic cholestasis, or involvement of large bile ducts leading to obstructive jaundice, has still been described.

It is biologically probable that the most frequent mechanism of liver dysfunction in multiple myeloma is related to the proteotoxicity from light chain production of the malignant plasma cells. This light chain production, within the bone marrow, may result in $\mathrm{AL}$ amyloid or monoclonal light chain deposition disease of liver. In addition, various antiplasma cell therapies employed for the treatment of myeloma can infrequently cause drug-induced liver injury. Although transfusion-related hepatic iron overload and clinically significant liver disease is a recognised complication in hematologic malignancies (particularly myelodysplastic syndrome) [1], transfusional iron overload has not been previously described in patients with multiple myeloma.

Correspondence to: Chakra P Chaulagain Email: CHAULAC@ccf.org

ecancer 2020, 14:1028

https://doi.org/10.3332/ecancer.2020.1028

Published: $30 / 04 / 2020$

Received: $27 / 11 / 2019$

Publication costs for this article were supported by ecancer (UK Charity number 1176307).

Copyright: $($ ) the authors; licensee ecancermedicalscience. This is an Open Access article distributed under the terms of the Creative Commons Attribution License (http:// creativecommons.org/licenses/by/3.0), which permits unrestricted use, distribution, and reproduction in any medium, provided the original work is properly cited. 
Herein, we describe a case of a 49-year-old female, with relapsed multiple myeloma, who presented with liver failure-due to both iron deposition and malignant plasma cell infiltration of liver-and subsequently died shortly after diagnosis. We also report on existing literature surrounding possible mechanisms of hepatic complications in multiple myeloma patients receiving treatments.

\section{Case presentation}

A 49-year-old female presented to the Emergency Department with profound fatigue, progressive jaundice and diffuse pruritus. Her medical history was significant for a 4-year history of relapsed, refractory international staging system stage III, IgG kappa MM. Previous treatments for MM included: 1) cyclophosphamide, bortezomib and dexamethasone (CyBorD), followed by 2) lenalidomide, bortezomib and dexamethasone, with consolidation hematopoietic stem cell transplantation after high dose melphalan $200 \mathrm{mg} / \mathrm{m}^{2}$ (MEL 200) and finally 3) lenalidomide regimen, initiated in 2013 , for maintenance.

The patient relapsed in 2016 and was initially treated with: 1) carfilzomib, pomalidomide and dexamethasone, followed by 2) daratumumab, pomalidomide and dexamethasone and finally 3) a regimen of bortezomib, dexamethasone, cisplatin, doxorubicin, cyclophosphamide and etoposide. Most recently, bendamustine and dexamethasone was used as a salvage regimen, employed as the last treatment, 4 weeks prior to the presentation.

In the several months prior to presentation, the patient had become transfusion-dependent, requiring both packed red blood cells and platelets, approximately twice per week. At the time of emergency admission, her vital signs were within normal limits. Her physical exam was remarkable for dry mucous membranes, profound jaundice with skin and scleral icterus, diffuse abdominal tenderness, and hepatosplenomegaly. Relevant laboratory findings are summarised in Table 1. She had normocytic anaemia with haemoglobin (10 g/dL), decreased platelets (40,000/mcL), elevated total bilirubin $(49.5 \mathrm{mg} / \mathrm{dL})$, elevated direct bilirubin ( $30 \mathrm{mg} / \mathrm{dL})$, normal aspartate transferase (AST) (18 U/L), elevated alanine transferase (ALT) $(51 \mathrm{U} / \mathrm{L})$, normal alkaline phosphatase $(80 \mathrm{U} / \mathrm{L})$, normal albumin $(3.8 \mathrm{~g} / \mathrm{dL})$, normal international normalised ratio (INR) (1.3), elevated ferritin $(15,037 \mathrm{ng} / \mathrm{mL})$ and elevated iron $(206 \mathrm{mcg} / \mathrm{dL})$-iron saturation and TIBC were not calculable.

Laboratory testing for hepatitis A, B and C, an autoimmune panel, haemolysis evaluation, and toxicology screen were unremarkable. An ultrasound of the liver demonstrated increased hepatic echotexture, but no biliary ductal dilation or hepatic nodules. A transjugular hepatic biopsy was performed to determine the etiology of liver dysfunction. The biopsy demonstrated infiltration of bile ducts and ductules by clusters of atypical plasma cells with features of plasmablasts (Figure $1 \mathrm{~A}$ and B). A trichrome stain highlighted peri-cellular fibrosis around the infiltrate (Figure 2A). In addition, increased deposits of iron in the hepatocytes and Kupffer Cells were noted, consistent with secondary iron overload (Figures 2B).

The observed liver failure was primarily attributed to malignant infiltration by relapsed myeloma and secondarily contributed by transfusional iron overload. The patient received symptomatic and supportive therapy with ursodeoxycholic acid, diphenhydramine and transfusions, as needed. Given her poor prognosis, goals of care were discussed, and she elected for palliative care/hospice. She died a week after the discharge from the hospital.

\section{Discussion}

Pathologic liver involvement, presenting concurrently with multiple myeloma at the time of diagnosis, is an uncommon finding. This type of presentation is often described in imaging studies as multiple nodular hepatic lesions mimicking metastatic tumours. It is often associated with poor prognosis, despite treatment efforts [2-4]. Infiltrative liver disease, due specifically to malignant infiltration by plasma cells, is also rare at the time of diagnosis, unless the presentation is due to an aggressive form of multiple myeloma manifesting as primary plasma cell leukemia $[5,6]$. 
Table 1. Laboratory results.

\begin{tabular}{|c|c|c|c|}
\hline Test name & Value & Normal Reference Range & Interpretation \\
\hline Haemoglobin & $10 \mathrm{~g} / \mathrm{dL}$ & $11.5-15.5 \mathrm{~g} / \mathrm{dL}$ & Low \\
\hline Platelet Count & $41 \mathrm{k} / \mathrm{uL}$ & $150-400 \mathrm{k} / \mathrm{uL}$ & Low \\
\hline ALT & $51 \mathrm{U} / \mathrm{L}$ & $7-38 \mathrm{U} / \mathrm{L}$ & High \\
\hline AST & $18 \mathrm{U} / \mathrm{L}$ & $13-35 \mathrm{U} / \mathrm{L}$ & Normal \\
\hline ALP & $80 \mathrm{U} / \mathrm{L}$ & $32-117 \mathrm{U} / \mathrm{L}$ & Normal \\
\hline Total Bilirubin & $49.5 \mathrm{mg} / \mathrm{dL}$ & $0.0-1.5 \mathrm{mg} / \mathrm{dL}$ & High \\
\hline Direct Bilirubin & $30 \mathrm{mg} / \mathrm{dL}$ & $0.0-0.2 \mathrm{mg} / \mathrm{dL}$ & High \\
\hline Albumin & $3.8 \mathrm{~g} / \mathrm{dL}$ & $3.5-5.0 \mathrm{~g} / \mathrm{dL}$ & Normal \\
\hline Iron & 206 & $41-186 \mathrm{ug} / \mathrm{dL}$ & High \\
\hline Ferritin & 15,037 & $13-150 \mathrm{ng} / \mathrm{mL}$ & High \\
\hline Transferrin* & Not calculated & Not calculated & $N / A$ \\
\hline Hepatitis A IgM & Negative & Negative & Normal \\
\hline Hepatitis B Surface Ag & Negative & Negative & Normal \\
\hline Hepatitis C Ab & Negative & Negative & Normal \\
\hline Acetaminophen & Undetectable & Undetectable & Normal \\
\hline INR & 1.3 & $0.8-1.2$ & Normal \\
\hline Creatinine & 0.4 & $0.58-0.96 \mathrm{mg} / \mathrm{dL}$ & Normal \\
\hline Calcium & 12.7 & $8.4-10.2 \mathrm{mg} / \mathrm{dL}$ & High \\
\hline SPEP M spike & $0.6 \mathrm{~g} / \mathrm{dL}$ & 0 & Abnormal \\
\hline LDH & 154 & $135-225 \mathrm{U} / \mathrm{L}$ & Normal \\
\hline Haptoglobin & 109 & $31-238 \mathrm{mg} / \mathrm{dL}$ & Normal \\
\hline Reticulocyte count & $1.4 \%$ & $0.4 \%-2.0 \%$ & Normal \\
\hline DAT & Negative & Negative & Normal \\
\hline
\end{tabular}

ALT: alanine transferase; AST: aspartate transferase; ALP: Alkaline phosphatase; Ag: antigen; Ab: antibody, ${ }^{*}$ Transferrin saturation is not reliable when ferritin levels $>1,200 \mathrm{ug} / \mathrm{L}$. LDH: lactate dehydrogenase, DAT: direct antiglobulin test, INR: International normalised ratio, SPEP: serum protein electrophoresis.

In contrast, diffuse hepatic infiltration, by malignant plasma cells, is much more frequent and has historically been described with an incidence rate up to $40 \%$ in an autopsy series before the discovery and use of novel agents for management of multiple myeloma [7]. Today, in the era of novel agents, the exact incidence of hepatic dysfunction, secondary to myeloma, is unknown. However, rather than direct hepatic plasma cell infiltration, the majority of cases reported in the literature are either secondary to hepatic amyloid deposition by systemic AL amyloidosis or, less commonly, due to monoclonal immunoglobulin light chain deposition disease of the liver [8-10].

Approximately, $10 \%-15 \%$ of multiple myeloma patients present with AL amyloidosis at the time of diagnosis, or will develop AL amyloidosis during the course of treatment $[11,12]$. Furthermore, liver involvement in patients with systemic AL amyloidosis is seen in approximately $25 \%$ of patients [11, 12]. Amyloidosis of the liver causes infiltrative liver disease, with associated elevation of alkaline phosphatase levels and hepatomegaly. However, in these cases, severe elevation of bilirubin and jaundice is uncommon. To determine liver involvement in patients with AL amyloidosis, liver size (as determined by ultrasound) greater than $15 \mathrm{~cm}$ in the absence of congestive heart failure, or alkaline phosphatase levels greater than 1.5 times the maximum normal values, can be used as disease indices [11, 12]. The diagnosis of amyloidosis is 
made by biopsy of an involved organ, or a surrogate site (e.g., abdominal fat pad, rectal mucosa or labial salivary glands)-subsequent visualisation of amyloid, with Congo red staining under polarised light, will demonstrate the characteristic apple-green birefringence. Isolated cases of jaundice, as a result of the obstruction of larger bile ducts by solid lesions from multiple myeloma, have also been described both at the time of diagnosis or at the time of relapse.

It is important to recognise that treatment-related factors may also play a role in liver dysfunction in patients with MM. For instance, anaemia is a common, concomitant finding in multiple myeloma. Additionally, anti-myeloma chemotherapy can cause severe anaemia, requiring red blood cell transfusion in some patients. This may be especially pertinent when myeloma becomes either refractory to treatment, or each remission period becomes shorter in duration as the disease becomes increasingly resistant to treatment. Transfusion-related iron overload, causing significant hepatic iron deposition, is a well-known complication in patients with myelodysplastic syndrome or haemoglobinopathies. However, red blood cell transfusion-related iron overload, causing hepatic iron deposition, is not yet well understood and has not been reported in multiple myeloma.

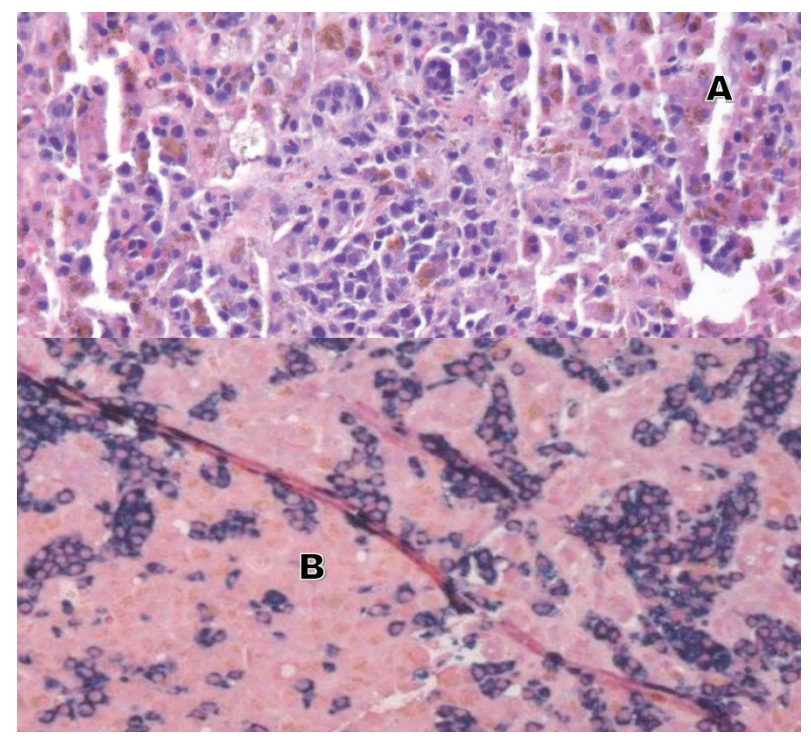

Figure 1. Liver biopsy (A and B). A: H\&E 20x, periductular clusters of malignant plasma cells with features of plasmablasts. In addition, increased iron deposits in hepatocytes and Kupffer Cells. B: kappa and lambda CISH 10x, plasma cells with monotypic kappa staining pattern.

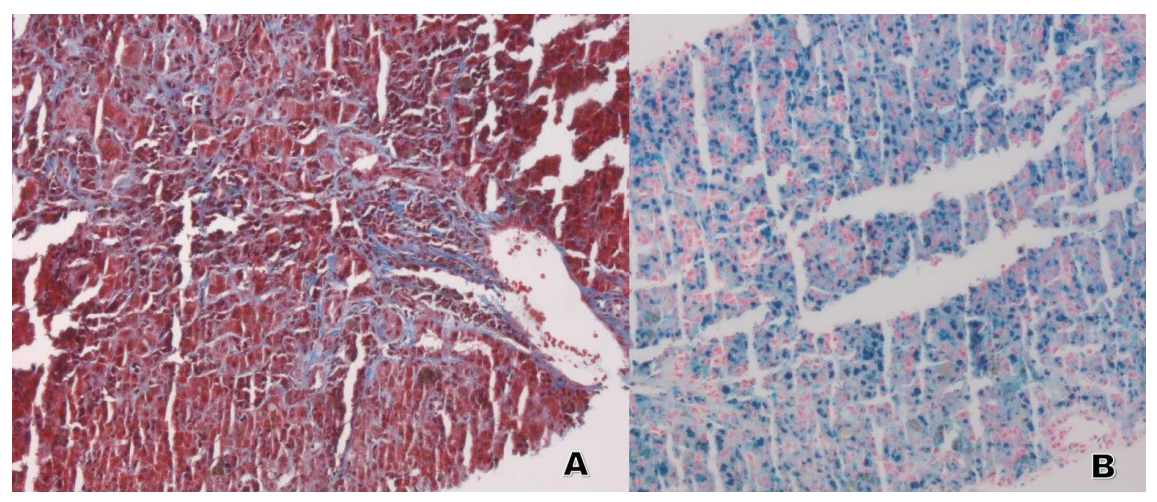

Figure 2. Liver biopsy. (A and B). A: Trichrome stain 10x: the stain highlights the presence of fibrosis around the malignant plasma cells. B: Iron Stain 10x: increased intracytoplasmic iron deposits in hepatocytes and Kupffer Cells. 
In this case report, it is believed that the frequent need for red blood cell transfusions, over the course of several months, contributed to the patient's hepatic iron overload. Unfortunately, due to absence of baseline iron studies and genetic testing for hereditary hemochromatosis (HFE gene), genetic predisposition to iron overload, due to underlying HFE gene mutation, cannot be definitively ruled out. To the knowledge of the authors, there is no current literature on the treatment of multiple myeloma in patients with known history of hereditary hemochromatosis, or secondary iron overload due to red blood cell transfusion. Therefore, the authors recommend collecting both baseline iron saturation and ferritin levels. If elevated ferritin and elevated iron saturation are found in patients with multiple myeloma, suspicions should be directed to transfusional iron overload or pre-existing hereditary hemochromatosis. A restrictive transfusional threshold, and the use of iron chelating agents, should be considered to prevent deposition of iron to visceral organs in patients with documented iron overload.

Treatment outcomes in multiple myeloma patients continue to improve with the use of immunomodulatory agents (IMiD) (e.g., thalidomide, lenalidomide and pomalidomide), proteosome inhibitors [(PI) e.g., bortezomib, carfilzomib, ixazomib], and more recently the use of immunotherapy (e.g., daratumumab and elotuzumab). Although rare, all approved IMiD therapies, including thalidomide, lenalidomide and pomalidomide, have been associated with severe hepatotoxicity during the treatment of multiple myeloma [13-15]. Similarly, all proteasome inhibitors have also been reported to exhibit an association with reversible liver dysfunction. For instance, bortezomib has been described to cause reversible liver dysfunction with liver function returning to normal after discontinuation [16]. Carfilzomib has been described to cause one non-fatal, but serious, case of liver failure, despite bortezomib and lenalidomide therapy being well-tolerated, previously [17]. Approximately, $6 \%$ of patients treated with ixazomib plus lenalidomide/dexamethasone, in relapsed/refractory multiple myeloma, exhibit liver dysfunction. As such, a reduced ixazomib dosage of $3 \mathrm{mg}$ (as opposed to standard $4 \mathrm{mg}$ ) is recommended in patients with existing moderate-to-severe hepatic impairment [18].

Immunotherapy represents a relatively new class of therapy available to treat multiple myeloma. Both daratumumab and elotuzumab have not yet been reported to be associated with serious hepatotoxicity or reactivation of latent hepatitis B viral infection. However, monoclonal antibody therapies, like rituximab, can cause serious hepatitis B virus reactivation, leading to fulminant hepatitis. Therefore, screening and management of latent Hepatitis B virus is recommended prior to rituximab therapy for B cell malignancies. Finally, physicians should also be aware of paraprotein interference in total bilirubin assays, which may lead to spuriously elevated total bilirubin, but normal levels of direct bilirubin in myeloma patients [19, 20]. Spurious hyperbilirubinemia from paraprotein interference may contribute to a clinical dilemma and lead to unnecessary testing. If artifactual elevation of total bilirubin is suspected, the laboratory should measure total bilirubin using alternative methods.

In conclusion, patients with myeloma and concomitant significant liver dysfunction, or liver failure, present difficulty in management. If the liver dysfunction becomes irreversible, delivering effective anti-myeloma therapy will not be possible, adversely affecting patient survival. Importantly, hepatotoxicity and rare cases of liver failure are potential consequences of chemotherapy in MM patients, even in patients who exhibit normal liver function tests upon diagnosis. Treatment options utilised to manage myeloma are generally considered hepato-safe, but in rare instances, may cause serious hepatotoxicity. Therefore, prompt recognition should be prioritised, as discontinuation of the offending agent can lead to recovery of liver function.

Plasma cell infiltration of the liver is a rare cause of death in the myeloma patient, either at the time of diagnosis or at the time of relapse. Aggressive presentation of multiple myeloma in the form of primary plasma cell leukaemia tend to cause liver involvement at the time of diagnosis; otherwise, liver involvement in myeloma is a late event in the course of the disease usually after multiple relapses or with development of secondary plasma cell leukaemia. Hepatic iron overload in this patient group can occur as a result of over transfusion. Therefore, monitoring for iron saturation and ferritin level are essential in order to better adopt restrictive transfusion practice and/or implement the use of iron chelation (in patients with documented iron overload) in order to mitigate iron deposition in the vital organs. Our patient was terminally sick at the time of presentation and was considered not a candidate for iron chelation therapy in the light of advanced relapsed myeloma with secondary liver involvement. Our patient presented with severe hyperbilirubinaemia and relatively normal liver enzymes. There was no evidence of intra-hepatic or extra-hepatic bile duct obstruction in ultrasound but microscopic bile ducts and ductules were infiltrated by malignant plasma cells causing intrahepatic microscopic cholestasis which must have played a central role in the severe hyperbilirubinaemia. The hepatic iron deposition may also have contributed to the degree of hyperbilirubinaemia. The presence of severe hyperbilirubinaemia with relatively normal liver enzymes in a myeloma patient may indicate malignant plasma cell infiltration of liver and iron deposition. 


\section{Conclusion}

Concurrent presentation of hepatic infiltration by myeloma and hepatic iron deposition is an uncommon event. This may be seen in a heavily pretreated relapsed myeloma patient with history of frequent transfusion. The presence of severe hyperbilirubinemia with relatively preserved liver enzymes levels and elevated iron saturation and ferritin may indicate malignant plasma cell infiltration of the liver and iron deposition. The diagnosis is established by biopsy. The prognosis is extremely poor as such patients are not candidates for iron chelation therapy, further antimyeloma therapy or liver transplantation.

\section{Acknowledgments}

The authors acknowledge the contributions of their patient and thank the Hematology malignancy research fund at Maroone Cancer Center of Cleveland Clinic Florida, Weston FL, USA.

\section{Conflicts of interest}

The authors declare that they have no conflicts of interest.

\section{Funding declaration}

There was no funding for this research.

\section{References}

1. Koreth J and Antin JH (2010) Iron overload in hematologic malignancies and outcome of allogeneic hematopoietic stem cell transplantation Haematologica 95(3) 364-366 https://doi.org/10.3324/haematol.2009.017244 PMID: 20207843 PMCID: 2833064

2. Wu XN, Zhao XY, and Jia JD (2009) Nodular liver lesions involving multiple myeloma: A case report and literature review World J Gastroenterol 15(8) 1014-1017 https://doi.org/10.3748/wjg.15.1014 PMID: 19248205 PMCID: 2653410

3. Marcon M, Cereser L, and Girometti R, et al (2016) Liver involvement by multiple myeloma presenting as hypervascular focal lesions in a patient with chronic hepatitis B infection BJR Case Rep 2(3) 20150013 PMID: 30459962 PMCID: 6243346

4. Tiu AC, Potdar R, and Arguello-Gerra V, et al (2018) Multiple liver nodules mimicking metastatic disease as initial presentation of multiple myeloma Case Rep Hematol 2018 Article ID 7954816 PMID: 29977630 PMCID: 5994292

5. Rahhal FE, Schade RR, and Nayak A, et al (2009) Hepatic failure caused by plasma cell infiltration in multiple myeloma World J Gastroenterol 15(16) 2038-2040 https://doi.org/10.3748/wjg.15.2038 PMID: 19399940 PMCID: 2675098

6. Bhandari MS, Mazumder A, and Vesole DH (2007) Liver involvement in multiple myeloma Clin Lymphoma Myeloma 7(8) 538-540 https:// doi.org/10.3816/CLM.2007.n.039 PMID: 18021472

7. Thomas FB, Clausen KP, and Greenberger NJ Liver disease in multiple myeloma Arch Intern Med 132 195-202 PMID: 4719547

8. Yamamoto T, Maeda N, and Kawasaki H (1995) Hepatic failure in a case of multiple myeloma-associated amyloidosis (kappa-AL) J Gastroenterol 30 393-397 https://doi.org/10.1007/BF02347517 PMID: 7647907 
9. Tabata S, Kurata M, and Takeda J, et al (2012) [Fatal hepatic failure due to AL amyloidosis in a patient with multiple myeloma]. Rinsho Ketsueki 53 1906-1910 PMID: 23257671

10. Macías Robles MD, Navia-Osorio García-Braga JM, and Menéndez Caro JL, et al (1994) [Jaundice secondary to intrahepatic deposit of light chains as a presenting form of multiple myeloma] An Med Interna 11 74-76 PMID: 8193237

11. Baker KR and Rice L (2012) The amyloidoses: clinical features, diagnosis and treatment Methodist Debakey Cardiovasc J 8(3) 3-7 https:// doi.org/10.14797/mdcj-8-3-3 PMID: 23227278 PMCID: 3487569

12. Chaulagain CP and Comenzo RL (2013) New insights and modern treatment of AL amyloidosis Curr Hematol Malig Rep 8(4) 291-298 https://doi.org/10.1007/s11899-013-0175-0 PMID: 24026941

13. Hanje AJ, Shamp JL, and Thomas FB, et al (2006) Thalidomide-induced severe hepatotoxicity Pharmacotherapy 26(7) 1018-1022 https://doi.org/10.1592/phco.26.7.1018 PMID: 16803426

14. Nojkov B, Signori C, and Konda A, et al (2012) Lenalidomide-associated hepatotoxicity-a case report and literature review Anticancer Res 32(9) 4117-4119 PMID: 22993370

15. Reed-Guy L, Hoteit MA, and Garfall AL (2018) acute liver failure associated with pomalidomide therapy for multiple myeloma Clin Lymphoma Myeloma Leuk 18(8) e337-e338 https://doi.org/10.1016/j.clml.2018.05.012 PMID: 29907543

16. Kim Y, Kim KY, and Lee SH, et al (2012) a case of drug-induced hepatitis due to bortezomib in multiple myeloma Immune Netw 12(3) 126-128 https://doi.org/10.4110/in.2012.12.3.126 PMID: 22916049 PMCID: 3422711

17. Kathi PR, Tama M, and Kundumadam S, et al A case of acute liver failure due to carfilzomib in multiple myeloma J Clin Pharm Ther 43(6) 918-920 PMID: 29969517

18. Kumar S, Moreau P, and Hari P, et al (2017) Management of adverse events associated with ixazomib plus lenalidomide/dexamethasone in relapsed/refractory multiple myeloma Br J Haematol 178(4) 571-582 https://doi.org/10.1111/bjh.14733 PMID: 28485007 PMCID: 5574012

19. Multiple Myeloma Interference in Total Bilirubin Customer bulletin 00-117 (Indianapolis: Roche Diagnostics) June 30, 2000. [Google Scholar]

20. Jang JY, Kim H, and Uh Y, et al (2013) Artifactual hyperbilirubinemia due to paraprotein interference in a case of multiple myeloma J Lab Med Qual Assur 35(2) 133-137 